\title{
Tegmen Tympani Cerebrospinal Fluid Leakage Repair: Transmastoid Approach Utilizing Stryker Bone Cement
}

\author{
Daniel Nguyen ${ }^{\mathrm{a}, \mathrm{c}}$, Jena Deitrick ${ }^{\mathrm{a}}$, William Sessions ${ }^{\mathrm{a}}$, Tam Nguyen ${ }^{\mathrm{b}}$
}

\begin{abstract}
Tegmen tympani defects resulting in cerebrospinal fluid (CSF) leakage into the middle ear can lead to hearing impairment, seizures, and meningitis. While these defects can be acquired in a variety of ways, they all require prompt surgical intervention. The majority are fixed utilizing a craniotomy through the middle cranial fossa (MCF). We present a case of a tegmen tympani defect successfully repaired by a transmastoid approach utilizing Stryker bone cement in combination with DuraMatrix, and advocate this surgical approach as an effective method for treating similar cases.
\end{abstract}

Keywords: Tegmen tympani; Cerebrospinal fluid; Leakage; Middle cranial fossa; Stryker bone cement; DuraMatrix

\section{Introduction}

The tegmen tympani is formed by the petrous and squamous portions of the temporal bone. It covers the semicircular canal and forms the roof of the tympanic cavity, separating the subarachnoid space from the middle ear. Tegmen tympani defects result in cerebrospinal fluid (CSF) leakage, and they can be acquired by a number of ways including recurring infection, surgery, trauma, congenital malformation, and spontaneous leakage [1]. The most common causes of CSF leakage by tegmen defects include acoustic neuroma or other middle ear surgery and chronic otological diseases, including cholesteatoma and neoplasms [1]. While extremely rare, congenital abnormalities such as Mondini's dysplasia and Hyrtl's fissure can also be reasons for leakages [1]. Spontaneous CSF leakage occurs with no history of trauma, surgery, or congenital abnormalities along with no clear explanation for the leakage [1].

Manuscript submitted January 23, 2018, accepted February 8, 2018

aSchool of Medicine, Texas Tech University Health Sciences Center, Amarillo, TX, USA

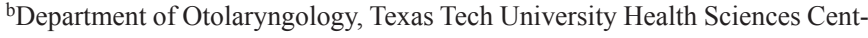
er, Lubbock, TX, USA

${ }^{\mathrm{c} C}$ Corresponding Author: Daniel Nguyen, School of Medicine, Texas Tech University Health Sciences Center, 1400 S Coulter St, Amarillo, TX 79106, USA. Email: DanielKNguyen92@gmail.com
The mastoid and epitympanum area are the most common sites of defect of the temporal bone resulting in CSF leakage. Mastoid area defects usually occur through trauma or iatrogenic causes while epitympanum area defects usually are spontaneous in nature [2]. These defects can lead to vertigo, hearing loss, CSF leakage leading to meningitis, facial paralysis, intracranial hemorrhage, and cerebral contusions [2]. Radiographic imaging techniques like head CTs combined with clinical presentation are used to make the diagnosis of a tegmen tympani defect. This condition always requires surgical intervention for treatment of symptoms and prevention of further complications.

\section{Case Report}

A 79-year-old female presented with otorrhea, which was subsequently diagnosed as tegmen tympani defect caused by failure of previous repair. Tegmen defect was seen on CT scan with CSF leakage in the middle ear, as shown in Figure 1. The patient was informed, consented, and taken to the OR for surgical repair. After the patient was prepped, facial nerve monitoring and lumbar drain was established. A posterior auricular incision was made to expose the mastoid cavity. The sigmoid sinus was identified but not entered. At this point, the old flap

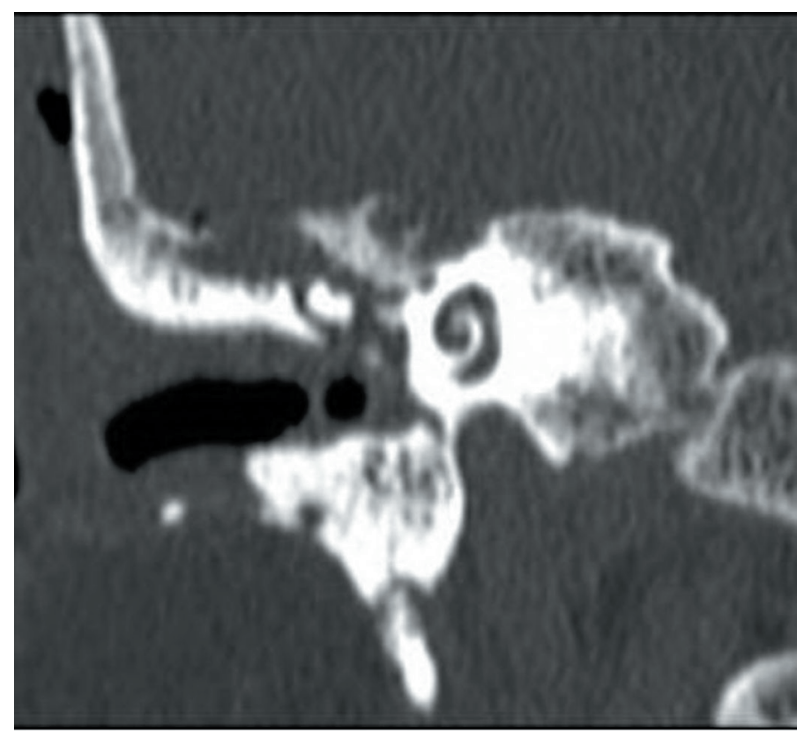

Figure 1. CT of the middle ear showing the tegmen defect. 


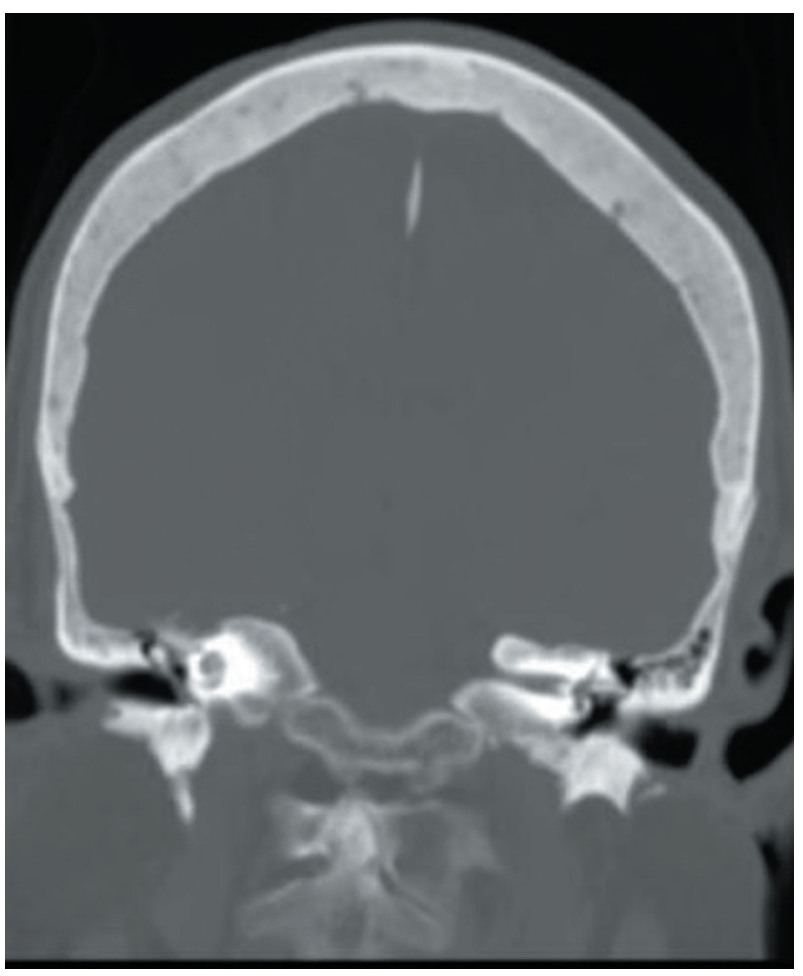

Figure 2. CT of the head shows lack of effusion in the middle ear 6 months postoperatively.

from a previous surgery for spontaneous CSF leak repair was removed. After removing some air cells with a small curette, the defect was clearly seen. There was a herniation of the dura and brain about $1.5 \times 1.5 \mathrm{~cm}$. The defect edges were cleaned, and the brain herniation was reduced and tucked under two layers of the DuraMatrix separated with Duragel. Stryker bone cement was then used underneath the tegmen from the lateral to the medial edge, near the short process of the incus/antrum. Bone cement was also used to peel out all the air cells as well at the lateral semicircular canal. The sigmoid was left intact to survey as a landmark in case of future mastoid surgery and for easy CT scan assessment. After closing, the lumbar drain was left in for a couple of days. After drain removal, the patient showed no sign of CSF leakage even after 6 months postoperatively seen in Figure 2.

\section{Discussion}

While the middle cranial fossa (MCF) approach is the most commonly used approach to fix tegmen defects, the transmastoid approach is gaining popularity due to its lower risk of complications. Some surgeons assert that the transmastoid approach cannot allow for adequate exposure to repair larger defects or those located medially or anteriorly, and removal of the ossicles may be required [3]. However, the MCF approach requires significant temporal lobe retraction, and is associated with more complications compared with other approaches $[1,3]$. The
MCF contains important neurovasculature, including divisions of the trigeminal nerve as well as the middle meningeal artery; these structures can be damaged during the procedure, resulting in bleeding or loss of facial sensation [4]. Therefore, the less invasive transmastoid approach is advantageous and does not require manipulation and elevation of the dura [4]. Recent literature has shown that both the MCF and transmastoid approaches have similar success rates, but there are not sufficient data to determine which approach is superior [3]. While we advocate for a transmastoid approach, we acknowledge that further research is necessary to adequately compare efficacy and complication rates among these two approaches.

Bone cement and DuraMatrix are commonly used materials well known for their utility in a variety of procedures, yet the use of them in concert is not reported within current literature. DuraMatrix consists of a flexible yet sturdy type I collagen membrane that can add mechanical strength to an injured dura mater, but it is absorbed within about 8 weeks of placement. Polymethylmethacrylate (PMMA) bone cement has been used in dental work, vertebroplasty and hip arthroplasties [5]. It is a grout like material supplements and strengthens bone interactions and provides long-term strength, especially against compressive forces [5]. When used synergistically, their different properties allow for the repair of the tegmen in a more effective manner.

The ideal approach for tegmen defect repairs must allow for successful repair while also minimizing complications. This case report demonstrated that a transmastoid approach using DuraMatrix and Stryker bone cement can effectively repair a tegmen defect while avoiding the risks of an MCF craniotomy.

\section{Disclosure}

This case study was approved by TTUHSC Research Integrity office.

\section{References}

1. Bento RF, Padua FG. Tegmen tympani cerebrospinal fluid leak repair. Acta Otolaryngol. 2004;124(4):443-448.

2. Patel A, Groppo E. Management of temporal bone trauma. Craniomaxillofac Trauma Reconstr. 2010;3(2):105113.

3. Lobo BC, Baumanis MM, Nelson RF. Surgical repair of spontaneous cerebrospinal fluid (CSF) leaks: A systematic review. Laryngoscope Investig Otolaryngol. 2017;2(5):215-224.

4. Braca JA, 3rd, Marzo S, Prabhu VC. Cerebrospinal Fluid Leakage from Tegmen Tympani Defects Repaired via the Middle Cranial Fossa Approach. J Neurol Surg B Skull Base. 2013;74(2):103-107.

5. Webb JC, Spencer RF. The role of polymethylmethacrylate bone cement in modern orthopaedic surgery. J Bone Joint Surg Br. 2007;89(7):851-857. 